experienced in the relief of pain from ulcers in the mouth. I would suggest that a trial of vitamin $B_{12}$ might be helpful in the early stages of peptic ulceration.

$$
\text { T. H. Oliver, M.D., F.R.C.P. }
$$

\section{Bronchial Adenoma}

Adenoma of the bronchus, a relatively rare condition occurring in approximately $6 \%$ of all bronchial tumours (Kramer and Som, 1935), is often not diagnosed for a considerable time after the onset of symptoms. The following case occurring in general practice is reported as a reminder of the disease and to record its unusual features.

\section{CASE RePort}

A housewife aged 67 was in good health until April 1952, when she developed a mild respiratory tract infection with an irritant cough, producing a symptom described by the patient as "like a bell ringing" in her chest. The illness resolved in 14 days with only symptomatic treatment. She became well and symptom-free, apart from slight loss of weight. Four months later (August) she developed a similar though more severe illness, with continued fever (maximum temperature $103^{\circ}$ F. $-39.4^{\circ}$ C.), irritant cough, and a little sputum. Physical signs of right lower zone bronchopneumonia developed. Her condition was not improved after a course of "sulphatriad," and her fever and physical signs resolved slowly during the next two weeks, after six daily injections of procaine penicillin, 300,000 units.

Chest $x$-ray examination as soon as she was ambulant, 18 days after the onset of symptoms, showed a rounded shadow adjacent to the right hilum, with no apparent peripheral collapse. There was no relevant past history or family history; she was an occasional smoker (one or two cigarettes a day).

On September 17 she was seen by Mr. O. S. Tubbs, who found no abnormal physical signs. Two weeks later, while awaiting admission for investigation, she suddenly collapsed in her home with acute pain in her right lower chest. On examination shortly afterwards she was found to be collapsed and dyspnoeic, her pulse was 90 , temperature $98^{\circ} \mathrm{F}$. $\left(36.7^{\circ} \mathrm{C}.\right)$, respirations 26. The $^{2}$ trachea was displaced to the left, and physical signs of a right pleural effusion were present. Chest $x$-ray examination confirmed the findings of collapse of the right middle and lower lobes, with an effusion.

She was admitted urgently to the Thoracic Unit. St. Bartholomew's Hospital, where diagnostic aspiration of the effusion in three places produced blood only. The sputum showed no tubercle bacilli, and grew only a scanty growih of Neisseria catarrhalis.

Bronchoscopy (Mr. O. S. Tubbs) showed : "Protruding out of the right middle lobe bronchus was a small palepink cherry-like tumour. The bronchoscope could be passed beyond this into the lower lobe, which appeared normal. Biopsy specimen taken ; rather profuse bleeding." The histological report on this specimen is of a "poorly differentiated adenocarcinoma."

At thoracotomy five days later a massive-2-3 pints (1-1.5 litres)-heavily blood-stained effusion was found, thought probably to be due to pleural carcinomatosis in view of the biopsy report and clinical picture presented. Accordingly, a right pleuropneumonectomy was performed.

The patient made an uninterrupted recovery, and was discharged from hospital 15 days later. She has remained well since.

It was subsequently evident from examination of the specimen that haemorrhage had occurred into the middle lobe behind the tumour, and that the middle lobe itself had ruptured, causing considerable haemorrhage into the pleural cavity. This undoubtedly occurred two weeks before her operation, and had caused her collapse in her home and her subsequent sudden deterioration at that time.

Pathology.- The tumour measured 7 by 6 by $5 \mathrm{~cm}$. The only remaining lung tissue of the right middle lobe was a portion of the medial segment and a thin peripheral zone stretched over the tumour mass. Anteriorly there was a rupture of the middle lobe and visceral pleura, with haemorrhage into the adjacent rim of lung tissue and into the pleural cavity. Histologically, the tumour was found to be projecting into the lumen of the bronchus, to have an adenomatous appearance, and to show no infiltration of deeper structures. The specimen was examined at the Institute of Diseases of the Chest, Brompton, London, and five different opinions were given : (1) Columnar-celled carcinoma of the bronchus ; (2) bronchial adenoma of lowgrade malignancy, or possibly secondary malignant change in pulmonary adenomatosis; (3) benign adenoma; (4) malignant adenoma; and (5) adenoma.

\section{COMMENT}

Adenoma of the bronchus is slightly commoner (62\%) in females (Foster-Carter, 1941). Symptoms may occur at any age, the average age of onset being 28 . Diagnosis is most commonly made during the fourth decade, an average of five years after the onset of symptoms (Foster-Carter, 1941). Cough, haemoptysis, and symptoms due to bronchial obstruction and its sequelae are predominant.

Radiological evidence of bronchial obstruction and/or the presence of an opaque tumour point to the diagnosis, which must then be confirmed by a bronchoscopic biopsy. The tumours are more commonly in the main bronchi, and therefore relatively accessible to the bronchoscope. Histologically. the precise nature of the tumour is often difficult to assess, and differences of opinion are common. The cells are usually uniform in shape and size; the cytoplasm is clear, and forms a fringe round the large deeply staining nucleus.

The tumours should be regarded as borderline between innocence and malignancy (Jackson and Norris, 1951). They may be locally invasive, and, very rarely, metastases have occurred through lymphatics (Adams et al., 1942). Malignant adenocarcinoma developing in an adenoma has been demonstrated histologically (Umiker and Storey, 1952).

Treatment of this potentially malignant condition varies from pneumonectomy, lobectomy, resection of the bronchus with end-to-end anastomosis (D'Abreu and MacHale, 1952) to piecemeal bronchoscopic resection, varying with each individual case. Opinion is now more in favour of exploratory thoracotomy and lobectomy as the operation of choice (Delarue, 1951). Radiotherapy is not of proved va'ue in treatment (Jackson and Norris, 1951).

I wish to thank Mr. O. S. Tubbs for permission to publish his case notes, and for his help and encouragement; Dr. G. J. Cunningham, senior lecturer in morbid anatomy, St. Bartholomew's Hospital ; and the Department of Pathology, St. Bartholomèw's Hospital, for the preparation of slides.

Michael A. Weller, M.B., B.S.

REFERENCES

Adams, W. E., Steiner, Paul E.. and Bloch, R. G. (1942). Surgery, 11,
5003.
D'Abreu. A. L., and MacHale, S. J. (1952). Brit. J. Surg., 39, 355.
Delarue. N. C. (1951). J. thorac. Surg. 21, 535.
Foster-Carter, A. F. (1941). Quart. J. Med.. 10, 139.
Jackson, Crevalier L., and Norris, Charles M. (1951). Laryngoscope,
St. Louis, 61, 238.
Kramer. Rudolph, and Som. Max L. (1935). Ann. Otol., St Louis, 44. 861.
Umiker, William, and Storey, Clifford F. (1952). J. thorac. Surg., 24. 120.

The United States Armed Forces Institute of Pathology is to collaborate with a group of Japanese pathologists headed by Dr. TORU MiYAI, professor of pathology at the Osaka University Medical School, in preparing a Japanese textbook on histopathology. The Institute will contribute about 300 standard photomicrographs to illustrate typical pathological conditions. The textbook is for use by Japanese medical students. Co-operation from the United States was requested for illustrations because of the difficulty of obtaining such material in the Far East. The project is expected to be completed within six months. 